\title{
Uma proposta para gerenciamento e preservação de imagens em medicina na EPM/Unifesp
}

\author{
Ana Paula Carrare \\ Mestre em informática em saúde pela Universidade de São Paulo. \\ E-mail: ana.carrare@fcmscsp.edu.br \\ Luiz Antonio Moura \\ Doutor em medicina. Professor da Universidade Federal de São Paulo \\ - Unifesp/EPM. \\ E-mail: luizmoura@dis.epm.br
}

Luiz Henrique Amaral

Doutor em astrofísica. Professor da Faculdade de Ciências Médicas da Santa Casa de São Paulo - FCMSCSP.

E-mail: luiz.amaral@fcmscsp.edu.br

\section{Daniel Sigulem}

Doutor em medicina. Professor Titular em informática em saúde da Universidade Federal de São Paulo - Unifesp/EPM.

E-mail: sigulem@dis.epm.br

\section{Resumo}

As imagens são muito utilizadas na área da saúde, tanto na prática profissional quanto na educação. Como conseqüência, há constante procura de formas para armazená-las, organizá-las e recuperá-las.O presente artigo descreve um ambiente desenvolvido com este intuito, e que permite aos profissionais compartilhá-las com outros membros da comunidade. Escolheu-se o formato digital, com interface web, no qual as imagens foram catalogadas com protocolo específico, indexadas a partir do texto descritivo e classificadas com o vocabulário controlado DeCs da Bireme. A consulta foi criada com base no modelo booleano, no uso de metadados e na pesquisa hierárquica. $O$ resultado da busca foi ordenado pela relevância de cada imagem. O produto final do trabalho foi denominado Biblioteca Virtual de Imagens em Medicina (BVIM) dispondo de áreas de acesso público e restrito, esta última utilizada como interface de administração e meio de alimentação do acervo pelos colaboradores.

\section{Palavras-chave}

Imagens em medicina. Biblioteca virtual. Biblioteca digital. Bancos de imagens. Preservação da informação.

\section{A proposal for the management and preservation of medicine images at the EPM/Unifesp}

\begin{abstract}
Images are very much used in the health area, both for the professional practice as for education. As a result, there is a constant search of ways to store them, organize them and retrieve them. The present article describes an environment developed for this purpose, which allows professionals to share them with other members of the community. For that purpose, a digital format with web interface was chosen, in which the images were catalogued with a specific protocol, indexed based on the description text and classified with the Bireme DeCs controlled vocabulary. The consultation was created based on the Boolean model, using metadata and hierarchical research. The result of the search was ordered according to the relevance of each image. The final product of the work was named Medicine Image Virtual Library (BVIM), making available areas of public and restricted access, the latter used as management interface and means for the cooperators to feed the image assets.
\end{abstract}

\section{Keywords}

Medical images. Virtual library. Digital library. Image banks. Preservation of information.

\section{INTRODUÇÃO}

A imagem tem estado presente em toda a trajetória da evolução da área de saúde e, atualmente, em um volume e importância muito maiores do que antes da descoberta do raio-X $(1895)^{1,2,3}$.

$\mathrm{Na}$ área de ensino, as imagens são utilizadas para demonstrar estruturas ou funcionalidades do corpo humano possíveis de serem mapeadas com as tecnologias existentes. Entre elas, há aquelas que despertam maior interesse, como as que representam casos raros, ou as que podem servir para revisões do que foi visto em atividades práticas. Outro exemplo é a digitalização de imagens obtidas a partir de lâminas com cortes histológicos, que possibilita ao aluno o contato com as mesmas sem o uso do microscópio.

A importância das imagens é incontestável nas práticas profissional ou de ensino ${ }^{4}$, justificando as muitas discussões no sentido de conseguir melhor forma de armazená-las, organizá-las e recuperá-las. Atualmente, em função da evolução tecnológica, muitas têm sido convertidas para o formato digital com a utilização de equipamentos apropriados (digitalizadores). Contudo, ainda existem muitas registradas no formato impresso, como papel, filme e diapositivo, que estão sujeitos à ação do tempo ou forma de armazenamento sem acondicionamento adequado.

O processo de digitalização não elimina os riscos de perda, mas pode se tornar uma das formas mais segura de preservação, desde que seja feito um planejamento prevendo a manutenção do acervo digital por longo período, levando em consideração as mudanças da tecnologia da informação ${ }^{5}$. Isso se aplica não só às imagens, mas também a outros tipos de informações.

Além de considerar a preservação, é necessário pensar na organização e recuperação. Estas, intimamente ligadas são também fundamentais, pois, se não houver boa organização, não poderá haver recuperação sem um dispendioso trabalho.

É muito freqüente encontrar imagens como parte de acervos pessoais de profissionais da saúde, em que cada um define uma forma de organização e recuperação 
(normalmente artesanal) sem qualquer preocupação com padrões já estabelecidos ${ }^{6}$. Isto tem sido um obstáculo para qualquer tentativa de unificação destes acervos e preservação do conhecimento neles contidos, começando pela dificuldade de reuni-los em um só lugar. Outra situação é o caso de imagens que fazem parte do prontuário de pacientes. Essas, em sua maioria, não são digitalizadas, o que muitas vezes provoca sua perda, por exemplo, pelo manuseio inadequado do prontuário ou acidentes no serviço de arquivos médicos (Same), bem como catástrofes naturais.

É importante, portanto, estudar e investigar os mecanismos de armazenamento e recuperação de imagens, assim como propor e avaliar as aplicações profissionais e educacionais para esta área.

Apesar de existirem muitas iniciativas com esse mesmo propósito, estas não têm sido suficientes, haja vista o número de imagens perdidas ou inacessíveis. Nesse contexto, foi desenvolvida a Biblioteca Virtual de Imagens em Medicina (BVIM), em plataforma web, buscando uma maneira de preservar e gerenciar imagens da área da saúde.

\section{OBJETIVO}

Desenvolvimento de um ambiente de organização e recuperação de imagens em saúde com foco na educação, que possa ser utilizado via web e que melhore a preservação e localização das imagens para os acadêmicos e profissionais da área, além de possibilitar acesso fácil e rápido por meio de uma metodologia específica e apropriada ao conhecimento destes. Também foi objetivo deste permitir aos profissionais organizar suas imagens e compartilhá-las com os outros membros da comunidade.

\section{BANCOS DE IMAGENS}

Os bancos de imagens são repositórios especializados que permitem aos indivíduos a realização de consultas. Atualmente, várias instituições e empresas são responsáveis ao menos por um.

Inicialmente eles ficavam disponíveis somente internamente nas instituições, mas as tecnologias web tornaram possível o acesso universal. Dentre os vários repositórios direcionados para a área de saúde e em plataforma web, foram selecionados alguns. A seguir, temos a relação de característica com numeração associada à tabela 1 .

1. Imagens tarifadas.

2. Contêm imagens de diversas subáreas.

3. O catálogo do acervo é composto de metadados utilizados por bibliotecas.

4. Consulta por:
a. diretório de assunto;
b. metadados, assunto e categoria;
c. metadados;
d. assunto;
e. metadados e assunto;
f. categoria baseada no vocabulário controlado MESH.

5. Resultado da consulta em forma de galeria.

6. As imagens são acompanhadas de informações específicas.

7. Geram lista com as imagens escolhidas para posterior download.

8. Acervo é composto de imagens especializadas.

9. Não são repositórios.

\section{TABELA 1}

Características dos repositórios pesquisados

\begin{tabular}{|c|c|c|c|c|c|c|c|c|c|c|c|c|c|c|}
\hline \multirow[t]{2}{*}{ Repositório } & \multirow[t]{2}{*}{1} & \multirow[t]{2}{*}{2} & \multirow[t]{2}{*}{3} & \multicolumn{6}{|c|}{4} & \multirow[t]{2}{*}{5} & \multirow[t]{2}{*}{6} & \multirow[t]{2}{*}{7} & \multirow[t]{2}{*}{8} & \multirow[t]{2}{*}{9} \\
\hline & & & & a & $\mathrm{b}$ & $\mathrm{c}$ & $\mathrm{d}$ & e & $\mathrm{f}$ & & & & & \\
\hline Medical Photographic Library? & $\mathrm{X}$ & $\mathrm{X}$ & $\mathrm{X}$ & & & $\mathrm{X}$ & & & & $\mathrm{X}$ & $\mathrm{X}$ & $\mathrm{X}$ & & \\
\hline Custom Medical Stock Photo ${ }^{8}$ & $\mathrm{X}$ & $\mathrm{X}$ & $\mathrm{X}$ & $\mathrm{X}$ & & & & & & $\mathrm{X}$ & $\mathrm{X}$ & $\mathrm{X}$ & & \\
\hline The Picture Source: science, medical and technical images ${ }^{9}$ & $\mathrm{X}$ & $\mathrm{X}$ & $\mathrm{X}$ & & & $\mathrm{X}$ & & & & $\mathrm{X}$ & $\mathrm{X}$ & & & \\
\hline Interesting Images ${ }^{10}$ & & $\mathrm{X}$ & $\mathrm{X}$ & $\mathrm{X}$ & & & & & & $\mathrm{X}$ & $\mathrm{X}$ & & & \\
\hline HonMedia: Images $^{11}$ & & $\mathrm{X}$ & & & & & & & $\mathrm{X}$ & $\mathrm{X}$ & & & & $\mathrm{X}$ \\
\hline University Library: Medical Images and Illustrations ${ }^{12}$ & & $\mathrm{X}$ & & $\mathrm{X}$ & & & & & & & & & & $\mathrm{X}$ \\
\hline History and Special Collections ${ }^{13}$ & & & $\mathrm{X}$ & & $\mathrm{X}$ & & & & & $\mathrm{X}$ & $\mathrm{X}$ & $\mathrm{X}$ & $\mathrm{X}$ & \\
\hline Images from the History of Medicine ${ }^{14}$ & & & $\mathrm{X}$ & & & & & $\mathrm{X}$ & & $\mathrm{X}$ & $\mathrm{X}$ & $\mathrm{X}$ & $\mathrm{X}$ & \\
\hline MedPix: Medical Image Database ${ }^{15}$ & & & & & & & $\mathrm{X}$ & & & $\mathrm{X}$ & $\mathrm{X}$ & $\mathrm{X}$ & $\mathrm{X}$ & \\
\hline Medical Images $^{16}$ & & & & & & & & & & & & & $\mathrm{X}$ & \\
\hline Images in Paediatric Cardiology $y^{17}$ & & & & & & & & & & & & & $\mathrm{X}$ & \\
\hline
\end{tabular}




\section{PRESERVAÇÃO DIGITAL}

A preservação digital consiste no planejamento e aplicação de recursos, métodos e tecnologias que garantam que a informação digital permaneça sempre disponíve ${ }^{5,6}$, independentemente das mudanças tecnológicas. A integridade da informação e a propriedade intelectual também devem ser resguardadas.

O desafio é real, pois os avanços tecnológicos acontecem rapidamente e dificultam a manutenção dos sistemas de arquivamento, que se não estiverem sempre atualizados podem implicar a perda da informação armazenada. Nesse aspecto, existem propostas para adoção de padrões ${ }^{5}$ que facilitem a migração, sempre que esta for necessária.

Mas, para que se ache uma solução, é necessário o comprometimento dos administradores dos repositórios, principalmente porque o custo é imprevisível.

\section{MATERIAIS E MÉTODOS}

No desenvolvimento do ambiente foram utilizadas linguagens de programação apropriadas para Internet HTML, PHP e Javascript -, e o banco de dados baseou-se em modelo relacional tendo como sistema gerenciador o MySQL ${ }^{\circledR}$ da MySQL Inc.

O sistema operacional instalado foi o Windows ${ }^{\circledR} \mathrm{XP}$ da Microsoft, e o servidor web utilizado foi o Apache $\AA^{\circledR} 1.3$ da Apache Inc versão para Windows. Durante a implementação e os testes técnicos, utilizou-se um computador Toshiba com a seguinte configuração: processador Pentium ${ }^{\circledR} \mathrm{IV}$ 1.7 Ghz da Intel, $512 \mathrm{Mb}$ de memória RAM, $40 \mathrm{~Gb}$ de disco rígido, monitor de 15", gravador de CD; e um digitalizador modelo Scanjet ${ }^{\circledR} 4400 \mathrm{C}$ da HP com resolução de até 1200 dpi.

Para a catalogação das imagens, foram utilizados um protocolo predefinido com dados pertencentes às imagens digitais $\mathrm{e}$ informações pertinentes aos padrões de registros do sistema de saúde. Os metadados são título, mídia original, espaço vetorial, dimensão, data de criação, método de obtenção, escala, descrição, padrão (colorido ou preto e branco), categoria (ilustração, radiologia ou patologia*), tipo (estática ou dinâmica).
A classificação das imagens foi desenvolvida para ser executada manualmente e utilizar o vocabulário controlado DeCs ${ }^{\circledR}$ da Bireme. A indexação das imagens foi feita com índice invertido ${ }^{18}$, gerado a partir das descrições da coleção à qual pertence a imagem ou do texto da sua própria descrição.

O mecanismo de busca foi desenvolvido com três opções: a primeira utilizando o método booleano, a segunda com pesquisa em campos de dados (metadados) da imagem e a terceira pela consulta hierárquica com base no vocabulário controlado citado anteriormente.

Para gerar o resultado da consulta, aplicou-se um método de ordenação que, a partir do cálculo do peso das palavras utilizadas determina as mais relevantes e, com base nestes pesos, coloca, no início da galeria recuperada, as imagens de maior relevância.

As imagens utilizadas nos testes foram de uma coleção de cortes histológicos, algumas foram submetidas a tratamento em programa específico para esta finalidade, o Photoshop ${ }^{\circledR} 5.5$ da Adobe Inc., no qual foram ajustados tamanho $(800 \times 600)$, cor, nitidez e brilho e removidas manchas não pertencentes às mesmas. Após os ajustes, elas foram armazenadas em formato JPG ou GIF*.

Para fins de demonstração, algumas imagens foram utilizadas na criação de pequenas aplicações desenvolvidas com o programa Flash $\AA 5.0$ da Macromedia Inc. Tais aplicações acompanham as respectivas imagens, quando essas são recuperadas pelo mecanismo de busca.

\section{ARQUITETURA DO AMBIENTE}

\section{FIGURA 1 \\ Arquitetura do ambiente}

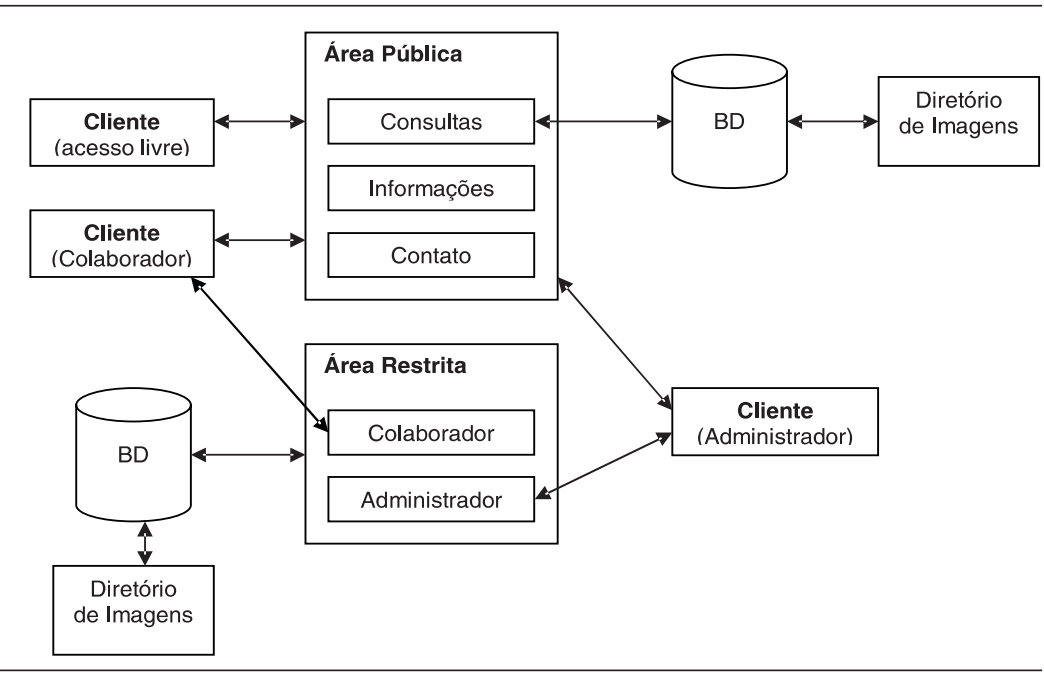

* Estas foram as categorias implementadas inicialmente.

* Esses formatos foram escolhidos por serem veiculados na Internet $\mathrm{e}$ mais familiares aos colaboradores. 


\section{RESULTADOS}

Este trabalho consistiu no desenvolvimento de um ambiente digital, que recebeu o nome de "Biblioteca Virtual de Imagens em Medicina (BVIM)", para gerenciar e recuperar imagens da área da saúde. Ele foi desenvolvido para ser acessível por meio de programas chamados de navegadores funcionando localmente, na intranet ou na internet.

O ambiente é composto de duas áreas: a pública e a restrita. A primeira permite a localização de imagens no acervo e a obtenção de informações referentes à BVIM e ao seu funcionamento. A segunda permite que os colaboradores contribuam com suas imagens ampliando o acervo. Ela também possibilita ao administrador o gerenciamento da BVIM. Esta divisão pressupõe a necessidade dos usuários abaixo descritos:

o público: pode consultar as áreas públicas da BVIM, inclusive, realizar busca de imagens no acervo;

o colaborador: tem acesso à área restrita por meio de identificação e senha, com o objetivo de fornecer imagens para o acervo, podendo utilizar esta área para gerenciamento das suas próprias imagens;

o administrador: também tem acesso à área restrita por meio de identificação e senha, sendo responsável pelo gerenciamento do ambiente, definindo quais imagens estarão ou não disponíveis publicamente.

A tela de abertura do ambiente (figura 2) foi dividida em duas partes por um menu horizontal. Na primeira, acima do menu, encontra-se uma imagem com o nome do ambiente e um formulário para digitação do usuário e senha da área restrita.

A segunda parte (abaixo do menu) contém links de acesso rápido para os itens já existentes no menu, e na lateral direita estão disponíveis dois tutoriais de apoio aos usuários do ambiente: Criação de Apresentações e Edição de Imagens.

A consulta ao acervo pode ser realizada de três formas: genérica, avançada ou hierárquica. Todas elas trazem como resultado uma galeria (figura 3) com imagens que, se clicadas, fornecem informações mais detalhadas sobre as mesmas (figura 4).
FIGURA 3

Galeria resultante da consulta

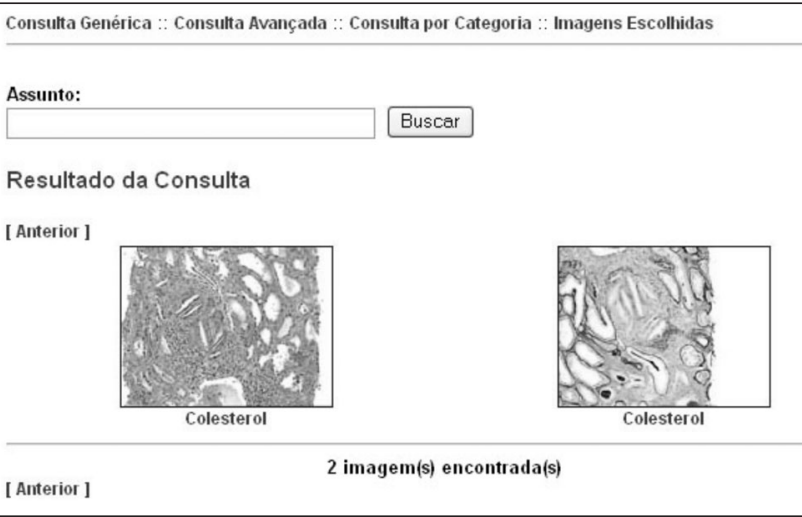

FIGURA 4

Detalhes específicos da imagem

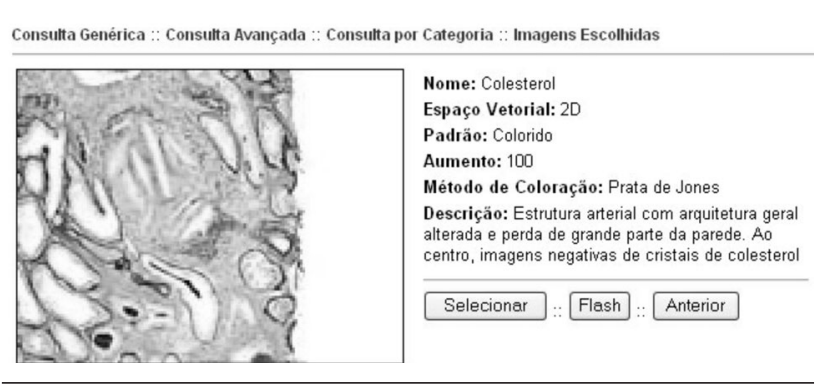


A área restrita pode ter duas visões: a do colaborador e a do administrador. No canto superior direito da tela principal (figura 2), o colaborador/administrador encontra um formulário para digitar sua identificação no ambiente e senha. Assim, poder ter acesso a sua área.

Na visão do colaborador, a interface é voltada para o gerenciamento das imagens. É nela que essas são inseridas no acervo.

A partir da tela principal, o colaborador pode gerenciar imagens relacionadas a casos clínicos ou a coleções didáticas.

Na visão do administrador, a interface é voltada para o gerenciamento do ambiente. Nela, é possível incluir ou excluir colaboradores, editar dados das coleções didáticas e classificar e indexar as imagens do acervo.

\section{DISCUSSÃO}

A BVIM foi implementada para ambiente web, de fácil acesso e independente de tecnologia proprietária. Pode, portanto, ser consultada de qualquer computador conectado à rede, o que a faz acessível a muito mais pessoas, 24 horas por dia.

Uma das razões que levou ao desenvolvimento da BVIM foi a constatação de que as imagens da área de saúde estão, de certa forma, restritas a determinados ambientes e grupos de profissionais e que os alunos das escolas de medicina têm acesso a elas somente por intermédio de seus professores. Dessas observações nasceu o formato da biblioteca: de um lado estão os usuários, que podem livremente realizar consultas no acervo; do outro os colaboradores, que são profissionais e/ou pesquisadores da área da saúde que fornecem as imagens para a BVIM.

Considerando tal formato, o colaborador tem um papel fundamental, pois é ele quem alimenta o acervo. Para estimulá-lo a fornecer suas imagens, uma área privativa lhe é destinada, na qual pode armazená-las juntamente com dados clínicos, laboratoriais e outras informações, bem como imagens que não tenham relação com casos clínicos, por exemplo, as consideradas didáticas ou históricas.

$\mathrm{Na}$ área destinada ao colaborador, a segurança é garantida pela identificação e uso de senha, como forma de preservar a privacidade das informações ali armazenadas. É importante ressaltar que um colaborador não tem acesso à área de outro. Isso também se aplica ao administrador do ambiente, que tem privilégios diferenciados, voltados somente para a manutenção do sistema.

\section{FIGURA 5}

\section{Tela principal do colaborador}

LHA, Bem vindo á área restrita

Casos Clinicos:

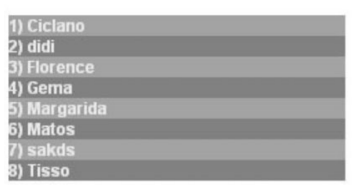

Coleções Didaticas:

Atualizar Novo Cadastro

\section{FIGURA 6}

Tela principal do administrador

\section{Colaboradores:}

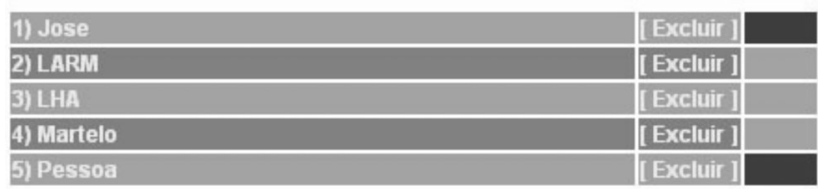

Atualizar Novo Colaborador Areas Atualizar DeCs

Ao armazenar as imagens em sua área, o colaborador concorda que estas podem ser vistas pelos usuários que realizam consulta no acervo. Para tanto, ao cadastrar-se, teve ciência de um termo de responsabilidade (cessão de imagens) que tem por objetivo evitar problemas com direitos autorais.

Nesse aspecto, a Medical Photographic Library ${ }^{7}$ tem uma filosofia parecida. Ela conta com colaboradores que são remunerados por imagem fornecida que for utilizada, ou seja, de alguma forma, em ambos os casos, eles ganham por colaborar com suas imagens.

Entretanto, apesar de o colaborador fornecer as imagens, não é ele que decide quais poderão ser recuperadas nas consultas públicas. Esse é um papel do administrador, que também as indexa e classifica.

A definição dos dados de paciente e exame foi orientada por profissionais médicos com base nas suas experiências. Atualmente, o ambiente permite a inserção de imagens de duas subáreas: da patologia e da radiologia, além da possibilidade de armazenar imagens gerais (ex. ilustrações). A implementação foi feita de forma a permitir que, em projetos futuros, possam ser incluídas novas áreas e subáreas da saúde, visto que as previstas obedecem a protocolos pertinentes a cada uma delas. 
Assim, o desenvolvimento deste módulo permite que cada subárea da saúde possa ter suas características respeitadas, pois foi percebido, mediante revisão de literatura, que isso constitui um dos impasses no desenvolvimento de sistemas de informação em saúde.

A maioria dos ambientes que se encontram em funcionamento contém imagens de várias áreas da saúde e de diversos tipos. Estas são catalogadas da mesma maneira que fotografias em bibliotecas tradicionais, ou seja, não há metadados específicos para cada área. Um dos poucos ambientes que se preocupa com essa diferenciação é o MedPix ${ }^{\mathrm{TM} 15}$ (especializado em radiologia), que trata da variação de metadados nesse escopo.

Na tabela 2, é possível observar os ambientes que trabalham ou não com imagens de várias subáreas. $\mathrm{Na}$ tabela 3, são apresentados os ambientes que têm especialização ou não dos metadados do catálogo.

A consulta ao acervo pode ser feita de três formas: genérica, avançada e por categoria, para que o usuário escolha o formato mais adequado para realizar suas pesquisas, podendo, inclusive, utilizar os três.

Na consulta genérica, ele informará a palavra que deseja, com a possibilidade de utilizar os operadores E e OU. O retorno será de imagens que contenham, em seu índice, as palavras utilizadas na consulta. Essa indexação é feita com base no texto da descrição da imagem e da coleção/ exame à qual ela pertence, gerando um índice invertido ${ }^{18}$, que, pela baixa quantidade de texto, não gera gasto excessivo de tempo na indexação; neste processo também é utilizada uma lista de stop word, para a retirada de palavras sem valor para o índice (pronomes, preposições, artigos etc.).

$\mathrm{Na}$ consulta avançada, ele preencherá os metadados solicitados no formulário e serão retornadas as imagens que contenham os mesmos dados informados pelo usuário.

$\mathrm{Na}$ consulta por categoria, serão recuperadas as imagens classificadas com o termo que o usuário clicou; estes termos são obtidos do vocabulário controlado DeCs.

Em relação às consultas, os ambientes comparados utilizam, individualmente, quatro tipos de consulta: por assunto, avançada (a partir do formulário com metadados específicos), por categoria e por diretório. Nas tabelas 4, 5, 6 e 7, podem ser vistas estas informações mais claramente.
TABELA 2

Comparativo entre os ambientes com relação à aceitação de mais de uma subárea da saúde

\begin{tabular}{|c|c|}
\hline Ambiente & $\begin{array}{l}\text { Aceita imagens } \\
\text { várias subáreas }\end{array}$ \\
\hline $\begin{array}{l}\text { BVIM; Medical Photographic Library }{ }^{7} \text {; Custom } \\
\text { Medical Stock Photo }{ }^{8} \text { HonMedia: Images }{ }^{11} \text {; } \\
\text { Interesting Images }{ }^{10} \text {; University Library: } \\
\text { Medical Images and Illustrations }{ }^{12} \text {; The Picture } \\
\text { Source: science, medical and technical }{ }^{9}\end{array}$ & Sim \\
\hline $\begin{array}{l}\text { Medical Images }{ }^{16} \text {; Images in Paediatric } \\
\text { Cardiology }{ }^{17} \text {; Images from the History of } \\
\text { Medicine }{ }^{14} ; \text { MedPix }{ }^{\mathrm{TM}} \text { : Medical Image } \\
{\text { Database }{ }^{15} \text {; History and Special Collections }}^{13}\end{array}$ & Não \\
\hline
\end{tabular}

\section{TABELA 3}

Comparativo entre os ambientes com relação à especialização dos metadados

\begin{tabular}{|c|c|}
\hline Ambiente & $\begin{array}{l}\text { Há variação de } \\
\text { metadados de } \\
\text { acordo com a } \\
\text { subárea }\end{array}$ \\
\hline BVIM; MedPix ${ }^{\mathrm{TM}}$ : Medical Image Database ${ }^{15 *}$ & Sim \\
\hline $\begin{array}{l}\text { Medical Photographic Library }{ }^{7} \text {; Custom } \\
\text { Medical Stock Photo }{ }^{8} \text {; HonMedia: Images }{ }^{11} \text {; } \\
\text { Interesting Images }{ }^{10} \text {; University Library: } \\
\text { Medical Images and Illustrations }{ }^{12} \text {; The Picture } \\
\text { Source: science, medical and technical }{ }^{9} \text {; Medical } \\
\text { Images }^{16} \text {; Images in Paediatric Cardiology }{ }^{17} \text {; } \\
\text { Images from the History of Medicine }{ }^{14} \text {; History } \\
\text { and Special Collections }{ }^{13}\end{array}$ & Não \\
\hline
\end{tabular}

* Há variação de metadados de acordo com a subárea da radiologia.

\section{TABELA 4}

\section{Comparativo entre os ambientes com relação à consulta} por assunto

\begin{tabular}{|c|c|}
\hline Ambiente & Permite \\
\hline $\begin{array}{l}\text { BVIM; History and Special Collections }{ }^{13} \text {; The } \\
\text { Picture Source: science, medical and technical } \\
\text { images }^{9} \text {; MedPix }{ }^{\mathrm{TM}} \text { : Medical Image Database }{ }^{15} \text {; } \\
\text { Images from the History of Medicine }{ }^{14}\end{array}$ & Sim \\
\hline $\begin{array}{l}\text { Medical Photographic Library }{ }^{7} \text { Custom } \\
\text { Medical Stock Photo } \text { Interesting Images }^{10} \text {; } \\
\text { University Library: Medical Images and } \\
\text { Illustrations }^{12} \text {; HonMedia: Images }{ }^{11}\end{array}$ & Não \\
\hline
\end{tabular}


TABELA 5

Comparativo entre os ambientes com relação à consulta avançada

\begin{tabular}{l|c}
\hline Ambientes & Avançada \\
\hline BVIM; History and Special Collections ${ }^{13} ;$ & \\
Medical Photographic Library ${ }^{7}$ Images from the & Sim \\
History of Medicine $^{14}$ & \\
\hline Custom Medical Stock Photo $^{8}$; Interesting \\
Images $^{10}$; University Library: Medical Images \\
and Illustrations $^{12}$; HonMedia: Images & ; The \\
Picture Source: science, medical and technical $^{11}$ & Não \\
images $^{9}$ : MedPix & \\
\hline
\end{tabular}

\section{TABELA 6}

Comparativo entre os ambientes com relação à consulta por categoria

\begin{tabular}{|c|c|}
\hline Ambientes & Por categoria \\
\hline $\begin{array}{l}\text { BVIM; History and Special Collections }{ }^{13} \text {; } \\
\text { HonMedia: Images }^{11}\end{array}$ & Sim \\
\hline $\begin{array}{l}\text { Medical Photographic Library }{ }^{7} \text {; Custom } \\
\text { Medical Stock Photo }{ }^{8} \text { Interesting Images }{ }^{10} \text {; } \\
\text { University Library: Medical Images and } \\
\text { Illustrations }{ }^{12} \text {; The Picture Source: science, } \\
\text { medical and technical images }{ }^{9} \text {; MedPix } \\
\text { Medical Image Database }{ }^{15} \text {; Images from the } \\
\text { History of Medicine }\end{array}$ & Não \\
\hline
\end{tabular}

\section{TABELA 7}

Comparativo entre os ambientes com relação à forma de consulta

\begin{tabular}{l|l}
\hline & \\
\hline BVIM; History and Special Collections & \\
Medical Photographic Library & ; HonMedia: \\
Images $^{11}$; The Picture Source: science, medical & Não \\
and technical images ${ }^{9}$ MedPix & : Medical \\
Image Database & ; Images from the History of \\
Medicine $^{14}$ & \\
\hline Custom Medical Stock Photo $^{8}$ Interesting & \\
Images $^{10}$; University Library: Medical Images & Sim \\
and Illustrations $^{12}$ & \\
\hline
\end{tabular}

O resultado de qualquer um dos tipos de consulta acima é uma galeria de imagens ordenada com base no cálculo de relevância das mesmas ${ }^{19}$. $\mathrm{O}$ usuário deve escolher a que lhe interessa (se houver). Ao clicá-la, ele poderá ver mais informações a seu respeito, com a possibilidade de copiá-la em seu computador. Veja nas tabelas 8,9 e 10, como os outros ambientes apresentam os resultados.

\section{TABELA 8}

Comparativo entre os ambientes com relação à geração de galeria

\begin{tabular}{|c|c|}
\hline Ambiente & Gera \\
\hline $\begin{array}{l}\text { BVIM; Interesting Images }{ }^{10} \text {; The Picture } \\
\text { Source: science, medical and technical images } \\
\text { MedPix }^{9 M} \text {; Medical Image Database }{ }^{15} \text {; Medical } \\
\text { Photographic Library }^{7} \text {; Custom Medical Stock } \\
\text { Photo }^{8} \text { Images from the History of Medicine }{ }^{14} \text {; } \\
\text { History and Special Collections }{ }^{13} \text {; HonMedia: } \\
\text { Images }^{* 11}\end{array}$ & Sim \\
\hline $\begin{array}{l}\text { Medical Images }{ }^{16} \text {; Images in Paediatric } \\
\text { Cardiology }{ }^{17} \text {; University Library: Medical } \\
\text { Images and Illustrations }{ }^{* 12}\end{array}$ & Não \\
\hline
\end{tabular}

* Não é repositório, ou seja, ele só localiza a imagem e aponta para sua origem, por isso as informações específicas e a lista de imagens dependem da origem das mesmas.

\section{TABELA 9}

Comparativo entre os ambientes com relação à apresentação de informações específicas da imagem recuperada

\begin{tabular}{|c|c|}
\hline Ambiente & Apresenta \\
\hline $\begin{array}{l}\text { BVIM; Interesting Images }{ }^{10} \text {; The Picture } \\
\text { Source: science, medical and technical images } \\
\text { MedPix }^{9} \text {; } \text { Medical Image Database }{ }^{15} \text {; Medical } \\
\text { Photographic Library }^{7} \text {; Custom Medical Stock } \\
\text { Photo }^{8} \text {; Images from the History of Medicine }{ }^{14} \text {; } \\
\text { History and Special Collections }^{13}\end{array}$ & Sim \\
\hline $\begin{array}{l}\text { Medical Images }{ }^{16} \text {; Images in Paediatric } \\
\text { Cardiology }{ }^{17}\end{array}$ & Não \\
\hline
\end{tabular}

\section{TABELA 10}

Comparativo entre os ambientes com relação à existência de lista de imagens escolhidas

\begin{tabular}{|c|c|}
\hline Ambiente & Contém \\
\hline $\begin{array}{l}\text { Interesting Images }{ }^{10} ; \text { The Picture Source: } \\
\text { science, medical and technical images } ; \\
\text { MedPix }{ }^{\mathrm{TM}} \text { : Medical Image Database }{ }^{15} ; \text { Medical } \\
\text { Images }^{16} ; \text { Images in Paediatric Cardiology }\end{array}$ & Sim \\
\hline $\begin{array}{l}\text { BVIM; Medical Photographic Library }{ }^{7} \text {; Custom } \\
\text { Medical Stock Photo } \text { P Images from the History }^{8} \\
\text { of Medicine }^{14} \text {; History and Special Collections }{ }^{13}\end{array}$ & Não \\
\hline
\end{tabular}


Ao realizar as comparações entre os ambientes, percebeuse que o de maior semelhança em objetivos foi o Images from the History of Medicine ${ }^{14}$; tecnicamente muitos são semelhantes, mas o grande diferencial da BVIM é sua filosofia, que torna seu formato mais democrático, pois, ao mesmo tempo que oferece aos profissionais uma forma de organizar suas imagens, faz com que ele compartilhe com outros o seu acervo.

\section{CONCLUSÕES}

O sistema preserva informações relevantes, em respeito às características de cada área ou subárea da saúde, e possibilita a organização e recuperação de imagens da mesma. Implementado para plataforma WEB, com áreas de consulta pública e restrita, satisfaz a necessidade de alunos e professores na busca por conhecimentos representados graficamente, sem comprometer a privacidade de dados do paciente.

Artigo submetido em 30/01/2006 e aceito em 13/03/2007.

\section{REFERÊNCIAS}

1. JOLY, M. Introdução à análise da imagem. Campinas: Papirus, 1996. p. 17-61. (Coleção Ofício da Arte e Forma).

2. QUÉAU, P. O tempo do virtual. In: PARENTE, A. (Org.). Imagem máquina: a era das tecnologias do virtual. 2. ed. Rio de Janeiro: 34 Literatura, 1996. p. 91-99. (Coleção Trans).

3. GALVÃO, P. B. A. Tecnologia e medicina: imagens médicas e a relação médico-paciente. Bioética, v. 8, n. 1, p. 127-36, 2000.

4. SHORTLIFE, E. H.; PERREAULT, L. E. Medical Informatics: computer applications in health care and biomedicine. 2 nd ed. New York: Springer-Verlag, 2000. p. 486-541.

5. MÁRDERO ARELLANO, Miguel Angel. Preservação de documentos digitais. Ciência da Informação, Brasília, v. 33, n. 2, 2004. Disponível em: $<$ http://www.scielo.br/scielo.php?script $=$ sci_arttext\&pid $=$ $\underline{\text { S0100-19652004000200002\&lng }=p t \& n r m=i s o}>$. Acesso em: 02 fev. 2007.
6. THOMAZ, K. P; SOARES, A. J. A preservação digital e o modelo de referência Open Archival Information System (OAIS). DataGramaZero, v. 5, n. 1, fev. 2004. Disponível em: <http:// www.datagramazero.org.br/fev04/Art_01.htm >. Acesso em: $02 \mathrm{fev}$. 2007.

7. THE WELLCOME TRUST. Medical photographic library. Disponível em: < http://medphoto.wellcome.ac.uk>. Acesso em: 15 jul. 2005.

8. CUSTOM medical stock photo. Disponível em: < www.cmsp.com/vlightbox/vlb12b1/welcome $>$. Acesso em: 15 jul. 2005.

9. THE PICTURE source: science, medical and technical images. Disponível em: <http://www.picsource.com.au/default.htm $>$. Acesso em: 15 jul. 2005.

10. MEDSTUDENTS. Interesting images. Disponível em: $<\underline{h t t p: / / ~}$ www.medstudents. com.br/image/image.htm $>$. Acesso em: $15 \mathrm{jul}$. 2005.

11. HEALTH ON THE NET FOUNDATION. HonMedia: images. Disponível em: < http://www.hon.ch/Media/media.html $>$. Acesso em: 15 jul. 2005.

12. KAROLINSKA INSTITUTET. University library: medical Images and Illustrations. Disponível em: <http://www.mic.ki.se/ MEDIMAGES.html>. Acesso em: 15 jul. 2005.

13. CALIFORNIA UNIVERSITY (UCLA). History and special collections. Disponível em: < http://www.library.ucla.edu/ biomed/his/ immi/>. Acesso em: 15 jul. 2005.

14. NATIONAL LIBRARY OF MEDICINE. Images from the history of medicine. Disponível em: <http://wwwihm.nlm.nih.gov/cgi-bin/ gw_44_3/chameleon?skin $=$ nlm\&lng $=\mathrm{en}>$. Acesso em: 15 jul. 2005.

15. AMERICAN COLLEGE OF RADIOLOGY. MedPix ${ }^{T M}$ : medical image database. Disponível em: <http://rad.usuhs.mil/medpix/ medpix.html?mode=news\#top $>$. Acesso em: 15 jul. 2005.

16. EUCALAB. Medical images. Disponível em: $<$ http://www.oeghmp.at/ eucalb/images_medical.html >. Acesso em: 15 jul. 2005.

17. GRECH, V. Images in paediatric cardiology. Disponível em: < http:// www.health.gov.mt/impaedcard/index.html> Acesso em: 15 jul. 2005.

18. BAEZA-YATES, R.; RIBEIRO NETO, B. Modern information retrieval. New York: ACM Press Books, 1999. p. 7-193.

19. BUCKLEY, C. et al. Automatic query expansion using SMART: TREC 3. In: TEXT RETRIEVAL CONFERENCE, 3., 1994, Gaithersburg. Proceedings... Gaithersburg: [s.n.], 1994. 